\title{
Le théorème de Skolem-Noether pour les modules sur des anneaux principaux
}

\author{
par Anne CORTELlA et JeAn-Pierre TIGNOL
}

RÉSUMÉ. Soit $k$ un anneau principal et $M$ un $k$-module de torsion de type fini. Nous donnons une preuve élémentaire du fait que tout automorphisme de $k$-algèbre de $R=\operatorname{End}_{k} M$ est intérieur.

ABSTRACT. Let $k$ be a principal ideal domain and $M$ a torsion $k$-module of finite type. We give an elementary proof of the fact that any $k$-algebra automorphism of $R=\operatorname{End}_{k} M$ is inner.

\section{Introduction}

D'après le théorème de Skolem-Noether classique, tout automorphisme de l'algèbre des endomorphismes d'un espace vectoriel de dimension finie sur un corps commutatif est intérieur. Ceci se généralise facilement aux automorphismes d'une algèbre centrale simple (voir par exemple [Thz, Th. $1.9 \mathrm{p} 4]$ ).

L'obstruction $\mathrm{SN} R=$ Aut $R / \operatorname{Int} R$ à cette propriété est connue pour différents types d'algèbre $R$, par exemple pour les algèbres d'Azumaya sur un anneau commutatif ([RZ]), l'obstruction étant alors un sous-groupe du groupe de Picard de l'anneau de base.

Pour des algèbres d'endomorphismes, la détermination de cette obstruction constitue un cas particulier de la question de Baer-KaplanskyWedderburn : quels peuvent être les isomorphismes d'algèbres, ou même d'anneaux, entre deux algèbres d'endomorphismes de modules (éventuellement même sur des anneaux distincts)? Les réponses les plus naturelles portent sur des modules libres ou projectifs, de type fini, sur des anneaux commutatifs (voir l'article d'état des lieux fait par A.V. Mikhalev [M]).

Nous nous intéressons ici à une autre sorte de modules ayant une notion de dualité : les modules de torsion de type fini sur un anneau principal. Grâce aux théorèmes de structure sur ces modules, on se ramène aisément à des modules fidèles de type fini sur un anneau local.

R. Baer en 1943 (dans [B1]) a montré que, modulo des hypothèses techniques supplémentaires mais en type non nécessairement fini, le théorème de Skolem-Noether vaut pour les algèbres d'endomorphismes de ce genre

Manuscrit reçu le 9 juillet 2004 . 
de modules. Sa démonstration utilise sa théorie ad-hoc des cycles sur l'ensemble partiellement ordonné des sous-modules du module de départ.

En 1952, I. Kaplansky ([K1],[K2]) donne une preuve complète du résultat de Baer, reposant sur une longue étude spécifique des anneaux fidèles sur un anneau local.

Nous donnons ici une preuve très courte du théorème de Skolem-Noether pour les algèbres d'endomorphismes de modules de torsion de type fini sur un anneau principal s'appuyant sur des résultats et des méthodes classiques sur les anneaux non commutatifs (voir par exemple [L1]).

Soit donc $k$ un anneau principal et soit $M$ un $k$-module de torsion de type fini, notons $R=\operatorname{End}_{k} M$. Dans une première partie, nous montrons qu'un automorphisme $\alpha$ de $R$ est intérieur si et seulement si $M$ et son tordu par l'action de $\alpha$ sont des $R$-modules isomorphes. Nous utilisons alors un système d'idempotents orthogonaux de $R$ pour décrire les modules projectifs de type fini sur $R$, ce qui, appliqué dans une troisième partie aux sous-modules de $p^{\ell}$-torsion de $M$ nous permet de conclure.

\section{Automorphismes d'algèbres d'endomorphismes}

Soit $M$ un module à droite sur un anneau commutatif quelconque $k$ et soit $R=\operatorname{End}_{k} M$. Par définition, $M$ est un module à gauche sur $R$. Comme $k$ est commutatif, les homothéties $h_{\lambda}: m \mapsto m \lambda$ pour $m \in M$ et pour $\lambda \in k$ fixé sont des endomorphismes du $k$-module $M$. On a donc un homomorphisme canonique $k \rightarrow R$ qui fait de $R$ une $k$-algèbre.

Soit $\alpha$ un $k$-automorphisme de $R$. Un procédé classique pour établir que $\alpha$ est intérieur consiste à tordre par $\alpha$ la structure de $R$-module à gauche de $M$. Définissons

$$
{ }^{\alpha} M=\left\{{ }^{\alpha} m \mid m \in M\right\}
$$

avec les opérations

$$
{ }^{\alpha} m+{ }^{\alpha} m^{\prime}={ }^{\alpha}\left(m+m^{\prime}\right) \quad \text { pour } m, m^{\prime} \in M
$$

et

$$
\alpha(r) \cdot{ }^{\alpha} m={ }^{\alpha}(r m) \quad \text { pour } r \in R \text { et } m \in M \text {. }
$$

Proposition 1.1. L'automorphisme $\alpha$ est intérieur si et seulement si $M \simeq{ }^{\alpha} M$ comme $R$-modules.

Démonstration. S'il existe $u \in R^{\times}$tel que $\alpha(r)=u r u^{-1}$ pour tout $r \in R$, alors l'application $\varphi: M \rightarrow{ }^{\alpha} M$ définie par

$$
\varphi(m)={ }^{\alpha}\left(u^{-1} m\right) \quad \text { pour } m \in M
$$

est un isomorphisme de $R$-modules car pour $m \in M$ et $r \in R$

$$
\varphi(r m)={ }^{\alpha}\left(u^{-1} r m\right)=\alpha\left(u^{-1} r u\right) \cdot{ }^{\alpha}\left(u^{-1} m\right)=r \cdot{ }^{\alpha}\left(u^{-1} m\right)=r \varphi(m) .
$$


Réciproquement, si $\psi:{ }^{\alpha} M \rightarrow M$ est un isomorphisme de $R$-modules, alors l'application $\psi_{0}: M \rightarrow M$ définie par

$$
\psi\left({ }^{\alpha} m\right)=\psi_{0}(m) \quad \text { pour } m \in M
$$

est un isomorphisme de $k$-modules, car pour $\lambda \in k$ la relation $\alpha\left(h_{\lambda}\right)=h_{\lambda}$ entraîne

$$
\psi_{0}(m \lambda)=\psi\left({ }^{\alpha}\left(h_{\lambda} m\right)\right)=h_{\lambda} \psi\left({ }^{\alpha} m\right)=\psi_{0}(m) \lambda \quad \text { pour tout } m \in M .
$$

Dès lors, il existe $u \in R^{\times}$tel que

$$
\psi_{0}(m)=u m \quad \text { pour tout } m \in M \text {. }
$$

Par la linéarité de $\psi$, on déduit

$$
\alpha(r) u m=\alpha(r) \psi\left({ }^{\alpha} m\right)=\psi\left({ }^{\alpha}(r m)\right)=u r m \quad \text { pour } r \in R \text { et } m \in M,
$$

donc

$$
\alpha(r)=u r u^{-1}
$$

\section{Structure de l'algèbre des endomorphismes des modules de longueur finie}

Lorsque $M$ est un $k$-module de longueur finie, l'algèbre $R=\operatorname{End}_{k} M$ est semi-primaire, c'est-à-dire que son radical de Jacobson $J$ est nilpotent et que le quotient $\bar{R}=R / J$ est (artinien) semi-simple, voir [L1, Th. de Hopkins-Levitzki, Th. 4.15, p. 59]. Pour reprendre le début de la preuve donnée en [L3, Ex. 21.24, p. 245], considérons une décomposition du $k$ module $M$ en $k$-modules indécomposables

$$
M=M_{1} \oplus \cdots \oplus M_{n}
$$

et désignons par $e_{i} \in R$ la projection $M \rightarrow M_{i}$ relative à cette décomposition. Les éléments $e_{1}, \ldots, e_{n}$ sont des idempotents orthogonaux qui satisfont

$$
e_{1}+\cdots+e_{n}=1
$$

et

$$
e_{i} R e_{i} \simeq \operatorname{End}_{k} M_{i} \quad \text { pour } i=1, \ldots, n .
$$

Comme $M_{i}$ est indécomposable de longueur finie, $\operatorname{End}_{k} M_{i}$ est un anneau local, par [L1, (19.17)]. Il en résulte par [L1, (21.18)] que l'image $\bar{e}_{i}$ de $e_{i}$ dans $\bar{R}$ engendre un idéal à gauche minimal de $\bar{R}$. La relation (2.1) entraîne

$$
\bar{R}=\bar{R} \bar{e}_{1} \oplus \cdots \oplus \bar{R}_{\bar{e}}
$$

donc $\bar{R}$ est artinien semisimple, et tout $\bar{R}$-module à gauche simple est isomorphe à l'un des modules $\bar{R} \bar{e}_{i}$. Quitte à renuméroter, on peut supposer que $\bar{R} \bar{e}_{1}, \ldots, \bar{R} \bar{e}_{r}$ sont non isomorphes deux à deux et représentent tous les types de $\bar{R}$-modules à gauche simples. 
Proposition 2.1. Pour tout $R$-module à gauche projectif de type fini $P$, il existe des entiers $n_{1}, \ldots, n_{r}$ déterminés de manière unique tels que

$$
P \simeq \operatorname{Hom}_{k}\left(M_{1}^{n_{1}} \oplus \cdots \oplus M_{r}^{n_{r}}, M\right) .
$$

Preuve (recopiée de [L1, (25.3)]). Comme $\bar{R}$ est artinien semi-simple, le $\bar{R}$ module $P / J P$ admet une décomposition unique

$$
P / J P \simeq\left(\bar{R} \bar{e}_{1}\right)^{n_{1}} \oplus \cdots \oplus\left(\bar{R} \bar{e}_{r}\right)^{n_{r}} .
$$

Une application simple du lemme de Nakayama (voir [L1, (19.27)]) permet de relever cet isomorphisme en

$$
P \simeq\left(R e_{1}\right)^{n_{1}} \oplus \cdots \oplus\left(R e_{r}\right)^{n_{r}} .
$$

Il suffit alors de remarquer que $\operatorname{Re}_{i} \simeq \operatorname{Hom}_{k}\left(M_{i}, M\right)$ pour tout $i=1, \ldots$, $r$.

\section{Modules de longueur finie sur un anneau principal}

Supposons à présent que l'anneau $k$ soit principal. Les $k$-modules indécomposables de longueur finie sont alors de la forme $k / p^{m} k$, où $p \in k$ est irréductible. Soit $M$ un $k$-module de longueur finie, de torsion $p$-primaire, et $R=\operatorname{End}_{k} M$. Pour tout entier $\ell>0$, soit $\ell M \subset M$ le sous-module de $p^{\ell}$-torsion,

$$
\ell M=\left\{m \in M \mid p^{\ell} m=0\right\} .
$$

Il est clair que $e_{\ell} M$ est stable sous tout endomorphisme de $M$, donc ${ }_{\ell} M$ est un $R$-module à gauche. Considérons une décomposition de $M$ en $k$-modules indécomposables

$$
M \simeq\left(k / p^{m_{1}} k\right) \oplus \cdots \oplus\left(k / p^{m_{n}} k\right) .
$$

Proposition 3.1. Pour $i=1, \ldots, n$, les $R$-modules $m_{i} M$ sont projectifs et indécomposables, et tout $R$-module à gauche projectif indécomposable de type fini est isomorphe à l'un des $m_{i} M$. En particulier, $M$ est un $R$-module indécomposable.

Démonstration. D'après la Proposition 2.1, les $R$-modules à gauche projectifs indécomposables de type fini sont les modules du type

$$
\operatorname{Hom}_{k}\left(k / p^{m_{i}} k, M\right) \simeq m_{i} M .
$$

Pour $m=\sup \left\{m_{1}, \ldots, m_{n}\right\}$, on a ${ }_{m} M=M$, donc $M$ est un $R$-module projectif indécomposable.

En combinant les Propositions 1.1 et 3.1, on obtient un analogue du théorème de Skolem-Noether pour les algèbres d'endomorphismes de modules de longueur finie sur un anneau principal. 
Théorème 3.1. Soit $M$ un module de longueur finie sur un anneau principal $k$. Tout $k$-automorphisme de $\operatorname{End}_{k} M$ est intérieur.

Démonstration. La décomposition primaire de $M$ induit une décomposition de $R=\operatorname{End}_{k} M$ qui est stable par tout $k$-automorphisme. On peut donc se limiter à considérer le cas où $M$ est de torsion $p$-primaire, pour un certain irréductible $p$. Alors $M$ est un $R$-module projectif indécomposable de type fini, d'après la Proposition 3.1. Si $\alpha$ est un $k$-automorphisme de $R$, le module ${ }^{\alpha} M$ est aussi projectif et indécomposable de type fini, car il est clair que l'opération qui à chaque $R$-module $P$ associe le module tordu ${ }^{\alpha} P$ préserve les modules libres et les sommes directes. De plus, la plus haute puissance de $p$ qui annule ${ }^{\alpha} M$ est la même que celle qui annule $M$. Dès lors, la Proposition 3.1 entraîne ${ }^{\alpha} M \simeq M$, et le théorème découle de la Proposition 1.1.

Remerciements : Nous remercions le FNRS qui subventionne partiellement le deuxième auteur, l' Université de Besançon qui a invité le deuxième auteur durant ce travail, et le réseau européen de K-théorie algébrique et groupes algébriques linéaires (HPRN-CT-2002-00287, KTAGS) qui a permis plusieurs rencontres entre les auteurs.

Nous tenons spécialement à remercier T.Y. Lam pour ses conseils de documentation sur ce sujet, qui ont largement contribué à l'état actuel de notre preuve, ainsi qu'à l'élaboration de la bibliographie ci-dessous.

Enfin, le premier auteur remercie Georges Gras de l'avoir accueilli à ses débuts au laboratoire de mathématiques de Besançon et pour tout ce qu'il lui a apporté au cours de sa carrière.

\section{Bibliographie}

[B1] R. BAER, Automorphism rings of primary abelian operator groups. Ann. Math. 44 (1943), 192-227.

[B2] R. BAER, Linear algebra and projective geometry. Academic Press (1952).

[F1] L. FUCHS, Infinite abelian groups, vol. 1. Academic Press (1970).

[F2] L. FUCHS, Infinite abelian groups, vol. 2. Academic Press (1973).

[G] R. Goebel, Endomorphism rings of abelian groups. Lecture notes in math. 1006 (1983), 340-353.

[I] I.M. ISAACS, Automorphisms of matrix algebras over commutative rings. Linear. Alg. Appli. 31 (1980), 215-231.

[K1] I. KAPLANSKY, Some results on abelian groups. Proc. Nat. Acad. Sci. USA 38 (1952), $538-540$.

[K2] I. KAPLANSKY, Infinite abelian groups. Univ. Michigan Press (1954); rev. ed. 1969.

[Kn] M.-A. Knus, Algebres d'azumaya et modules projectifs. Commen. Math. Helv. 45 (1970), 372-383.

[L1] T.Y. LAM, A first course in non-commutative rings. Springer-Verlag (1991).

[L2] T.Y. LAM, Modules with isomorphic multiples and rings with isomorphic matrix rings, a survey. Monographies de l'enseign. math., Genève 35 (1999). 
[L3] T.Y. LAM, Exercises in Classical Ring Theory. Springer-Verlag (1995).

[M] A.V. MIKHALEV, Isomorphisms and anti-isomorphisms of endomorphism rings of modules. Proc. first international Tainan-Moscow alg. workshop, Berlin (1996).

[RZ] A. Rosenberg, D. Zelinsky, Automorphisms of separable algebras, Pacif. J. Math. 11 (1961), 1109-1117.

[Thz] J. Thevenaz, G-algebras and modular representation theory. Oxford Sc. Publi. (1995).

[W] K.G. Wolfson, Anti-isomorphisms of endomorphism rings of locally free modules. Math. Z. 202 (1989), 1951-1959.

Anne CoRTELla

UMR CNRS 6623, Laboratoire de Mathématiques

Université de Franche-Comté

16 route de Gray

F-25030 Besançon Cedex, France

E-mail : cortella@math. univ-fcomte.fr

Jean-Pierre TIGNOL

Département de Mathématiques

Université Catholique de Louvain

B-1348 Louvain-la-Neuve, Belgique

E-mail : tignol@math.ucl.ac.be 